\title{
Iterative Design of A Braille Writing Tutor to Combat Illiteracy
}

\author{
N. Kalra, T. Lauwers, D. Dewey, T. Stepleton, and M. B. Dias
}

\begin{abstract}
Less than 3\% of the 145 million blind people living in developing countries are literate. This low literacy rate is partly due to the lack of trained teachers and the challenges associated with learning to write Braille on a traditional slate and stylus. These challenges include writing from right to left, writing mirrored images of letters, and receiving significantly delayed feedback. Extensive conversations with the Mathru School for the Blind near Bangalore, India, revealed the need for a robust, low-power, low-cost Braille writing tutor. We present an iterative and participatory design process resulting in the creation and refinement of a prototype Braille writing tutor system. This system uses a novel input device to capture a student's activity on a slate using a stylus and uses a range of techniques to teach Braille writing skills to both beginner and advanced students. We report on lessons learned from the implementation of this project and from a six-week pilot study at the Mathru school, and outline future directions for improvement.
\end{abstract}

Index Terms - Developing nations, intelligent tutoring systems, user-centered design

\section{INTRODUCTION}

More than $90 \%$ of the world's 161 million blind and visually impaired people live in developing communities [1]. Despite the importance of literacy to employment, social well-being, and health, the literacy rate of this population is estimated at below 3\% [2]. Braille, the primary method of reading and writing for the blind, is a tactile system in which embossed dots representing letters, symbols, and numbers can be read with the fingers. A Braille letter is formed by embossing some subset of six dots arranged in a $3 \times 2$ cell. Figure 1 shows schematics of a Braille cell and a photograph of a page of Braille. For the blind, literacy in Braille is often the key to independence in home and at work [3]. It is said that the system has "liberated a whole class of people from a condition of illiteracy and dependency and has given them the means for self-fulfillment and enrichment" [4].

Despite the advantages that Braille literacy imparts, there are a number of barriers to learning Braille in developing countries. According to the Mathru Educational Trust for the Blind, the main barrier in India's case is limited opportunities

Manuscript received on August 30, 2007. This work was funded by TechBridgeWorld's V-Unit program, the IFYRE program, and the National Science Foundation's IGERT fellowship in assistive technology (DGE-0333420).

N.Kalra is with the RAND corporation, nkalra@rand.org

T. Lauwers is with Carnegie Mellon University, tlauwers@andrew.cmu.edu, 412-268-6723

D. Dewey is with Carnegie Mellon University, danieldewey@cmu.edu

T. Stepleton is with Carnegie Mellon University, tss@ri.cmu.edu

M.B. Dias is with Carnegie Mellon University, mbdias@ri.cmu.edu

Note that this worked was performed while N.Kalra was a Ph.D student at Carnegie Mellon. for education because parents and families of blind children often do not realize the possibility or value of educating their child. Even when the desire to educate is present, children may not receive sufficient guidance at home or in traditional schools because very few people are trained to teach Braille. Unfortunately, poorer areas tend to have both a disproportionately high number of blind people [1] and fewer resources for educating them.

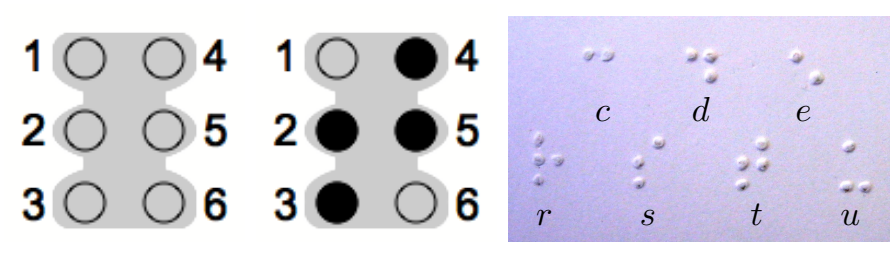

Fig. 1. A schematic of a Braille cell (left) and the letter ' $t$ ' (center). The black circles represent embossed dots while the light grey circles represent unembossed dots. A sample of Braille (right).

Furthermore, the traditional method of writing Braille itself creates formidable challenges to literacy. In developed countries, Braille is usually embossed with a six-key typewriter known as a Brailler; these devices are fast and easy to use but also cost over US\$600 each [5]. In developing countries, such devices are prohibitively expensive and Braille is almost always written with a slate and stylus as shown in Figure $2^{1}$. Using these tools, Braille is written from right to left so that the page can be read from left to right when it is removed from the slate and turned over. For blind children, learning to write Braille in this manner can be difficult. First, children must learn mirror images of all letters which doubles the alphabet and creates a disparity between the written and read forms of each letter. Second, feedback is delayed until the paper is removed and then flipped over and read. For young children, this delay can make Braille conceptually challenging since the act of writing has no discernible, immediate effect. It also takes longer for both the student and the teacher to identify and correct mistakes and this slows learning. Finally, even the thick paper used to write Braille may be expensive or in limited supply [6]. The work presented focuses primarily on aiding student learning of Braille writing by improving the feedback provided to the learner.

We are presenting the results of a collaborative design process with the Mathru Educational Trust for the Blind

\footnotetext{
${ }^{1}$ Reprinted with permission from LightHouse for the Blind and Visually
} Impaired. 


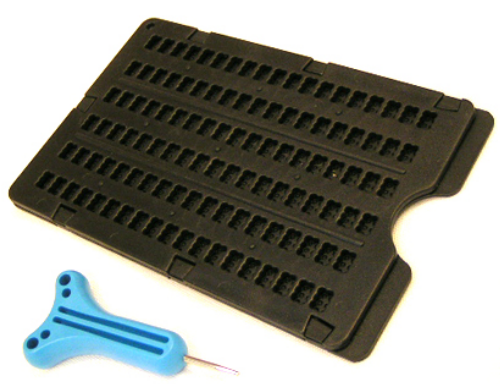

Fig. 2. A Braille slate and stylus.

near Bangalore, India, a non-profit, organization dedicated to educating and rehabilitating the visually-impaired. As a result of discussions with the administration, teachers, and students at Mathru, we believe there is great potential to address these difficulties using an intelligent tutor. To this end, we have created the Braille Writing Tutor shown in Figure 3 which consists of a low-cost, low-power electronic slate and stylus that mimics a regular slate and stylus and interfaces with a computer running the tutor software. Although it would have been possible to create a tutor which teaches Braille using a normal keyboard, we specifically decided to target the skill of writing Braille with the slate and stylus, such that students are not dependent on potentially difficult to access computer technology in their post-school lives. The tutor monitors a student's writing and provides immediate audio feedback using text-to-speech synthesis that is tailored to the skill level of the learner and both highlights new concepts and reinforces skills that the student already has. It can also act as a diagnostic tool to help identify some challenging areas for students.

This paper describes the techniques and process used to design and refine the Braille Writing Tutor. We describe related work on collaborative design, automated tutors, and teaching aids for the blind, the initial genesis of the idea, development of the first prototype, lessons we learned from testing the prototype at Mathru, and how we redesigned the tutor based on those lessons. We conclude with a discussion of the Braille Writing Tutor and an outline of future research directions.

\section{RELATED WORK}

Researchers recognize the importance of deep involvement of end-users in the design process when creating interactive technology, especially when the technology is targeted to developing communities whose cultural and social needs may be quite different from those of the technology designers [7]. There are several methods for involving end-users in the design process; rapid ethnography allows researchers to quickly test and iterate a product in-situ with end-users [8]. Participatory research goes further, with collaboration between researchers and users beginning during the initial stages of the design process, and by moving control over the direction of research closer to those who will eventually apply the research [9]. Although not a pure case study of these methods, our emphasis on collaboration and in-situ development express elements of both participatory research and rapid ethnography.

A number of intelligent tutors exist for a range of subjects and skills including math [10], English reading [11], speaking [12], and computer programming [13]. Encouragingly, many of these tutors have achieved success in the classroom. Nevertheless, they have limited impact on our goals for a number of reasons. Firstly, of course, they are not tailored to writing. Secondly, they require sight and use written instruction extensively in the tutoring process; in contrast, a tutoring system for the blind usually depends heavily on audio feedback. Thirdly, this limitation means that most existing automated tutoring systems for the blind are fairly simple (e.g. the Talking Braille Tutor $囚$ teaches only individual symbols [14]) and cannot teach complex skills such as writing using a slate and stylus. A notable exception is the Speech Assisted Learning (SAL) device which tutors reading and math using a stand-alone refreshable Braille display [15]. Fourthly, like SAL (which costs US \$4600) and the Talking Braille Tutor (which costs US \$300), most assistive technology is prohibitively expensive because the number of blind people who could potentially access it is very small. An exception is the low cost Sparsha system [16] which is a software package specifically designed for the blind in India, supporting English and a dozen native Indian languages. With Sparsha, a blind user can type on either a normal keyboard or a novel input device [17] which uses the six-key modality of writing Braille, and translate the Braille to text in any of the supported languages. As such, the Sparsha system complements the Braille Tutor nicely, with the former improving the experience of writing Braille on a computer and the latter teaching manual Braille writing skills. We believe that the LISTEN English reading tutor [11] which listens to children read aloud and provides audio feedback has the most relevance to our work as it uses an alternate medium of interaction (spoken words) and teaches a basic literacy skill (reading). Moreover, it employs many of the Artificial Intelligence techniques we intend to additionally incorporate into our tutor. Nevertheless, the need remains for a writing tutor specifically tailored to meet the needs of the blind in developing countries.

\section{IDEATION}

The idea of the Braille writing tutor was conceived through extensive discussions with teachers from the Mathru School for the Blind in Bangalore, India, our partner in this collaborative design process. The Mathru School is residential and currently has 44 children enrolled in grades one to six. In addition to providing the standard curriculum for the state of Karnataka, Mathru teaches daily living skills such as mobility and food preparation, offers vocational training such as computer classes, provides medical care, and encourages talent, personality development, and self-confidence. Six of the eight teachers at Mathru are themselves blind or visually impaired. Additionally, most of Mathru's students come from the very poorest of Karnataka's villages where they may previously 
have had no access to running water or electricity, much less computers and electronics.

The concept of the Braille Tutor came about through a unique, non-traditional dialogue with Mathru. We approached Mathru knowing that blindness can create extreme life challenges for those in developing countries, but without a clear picture of the specifics. The teachers at Mathru were intimately familiar with those challenges but were unaware of how technology could help. Therefore, we began by asking Mathru for a laundry list of all the difficulties their students faced, from education to personal care to food to transportation. Their list included being unable to determine whether water was clean or dirty, but, to our surprise, pointedly did NOT include things like having difficulty playing team games such as cricket. The list revealed that reading and writing were problematic for young children, and, to investigate further, we requested photos and videos of their students writing Braille at different levels. From these videos we identified the writing difficulties mentioned earlier in this paper (many of which were never specifically articulated by teachers or students) and realized that technology could play a role in mitigating these challenges. We developed the Braille Tutor concept through dialogue with researchers at Carnegie Mellon University and blind adults in the Pittsburgh area and through continued discussions with Mathru. This process underscored that thoroughly understanding user needs is critical to a successful solution, but also revealed that this understanding can come through loosely focused discussions (e.g. lists of wants, videos) and need not be precisely articulated by the user.

\section{A. Design Goals}

Once the idea of the Braille Tutor was conceptualized we came up with a list of design goals for both hardware and software based on our conversations with Mathru which we felt were necessary to meet for the project to be successful. These included:

- Low-Cost Unlike other tutors, ours must be affordable to members of the base of the economic pyramid who live on less than US\$2 a day. We hope to make it affordable to every village or rural school even if it cannot be affordable to individuals. Our target price is US $\$ 20$ per unit for systems requiring an external computer and US $\$ 40$ per unit for systems with embedded text-to-speech hardware that can operate without a computer.

- Low-Power In developing countries, electricity may be unreliable, in limited supply, or simply unavailable. The tutor must maximize the resources available, be robust to unreliable power, and be able to be powered by alternative sources. Our target power consumption is $300 \mathrm{~mW}$, or enough to operate for about 50 hours on 4 AA batteries.

- Robust The tutor's hardware components must be rugged enough to be extensively used and abused by students for a long time.

- Easily Operated The tutor must be easily and independently operated by a blind person. This means that both the hardware and the software must be accessible to someone with little or no computer literacy or experience with electronics. It must also provide guidance that can be utilized without the presence of a teacher.

- Easily Understood The tutor's speech module must be understandable given the age and background of the learner. Depending on the circumstances, it may use local languages, local dialects, and age-appropriate voices.

- Locally Maintainable The tutor must be designed with easily available components so that if any of the electronic components fail, repairs can be made on-site or nearby. This means using commonly available materials and manufacturing techniques.

- Transferable Learning Students learning writing with the tutor must be able to transfer this learning to a regular slate and stylus. Therefore the tutor experience must be made as similar to using the slate and stylus as possible.

\section{First E-Slate DESIGN}

We designed the first version of the E-Slate over a six month period at Carnegie Mellon University; the design and assembly of a field-testable prototype constituted a V-Unit, an independent study course offered through the TechBridgeWorld initiative [18]. The E-Slate was redesigned four times; after each iteration we gathered feedback from local engineering and human-computer interaction communities as well as from Mathru and made improvements. As an example, one major improvement was the addition of a second line of Braille cells in the input area after teachers at Mathru indicated that switching rows was one of the most difficult concepts for students to grasp.

The most challenging aspect of the design of the E-Slate was to match the use experience as closely as possible to that of a regular slate and stylus while still meeting our low-cost and low-power goals. As shown in Figure 3, the input area of the E-slate consists of two rows of 16 Braille cells each and is integrated directly into the circuit board to maximize robustness and minimize cost. A cutout from a normal plastic slate is placed over top of the two Braille rows to give students the exact same feel as when writing on a standard slate. We used an extremely low-cost and low-power microcontroller, the Atmel ATMEGA88 [19] in conjunction with a custom resistor network decoding circuit to handle the sensing of stylus location in the input area. If the stylus is in contact with any dot in the input area, the Atmega88 senses which dot in which cell the stylus is contacting and transmits the information to a computer over the serial port. The stylus is a standard Braille stylus modified to connect it to the slate via a wire soldered to its metal tip.

A small speaker and four buttons provide a basic interaction modality between the student and the E-slate, even when it is not connected to a computer. The speaker emits a tone whenever the stylus makes contact with a dot in a Braille cell, with each dot being mapped to a different musical note. The four buttons activate and deactivate several features. Button 1 toggles the heartbeat LED, which was used by us as a 


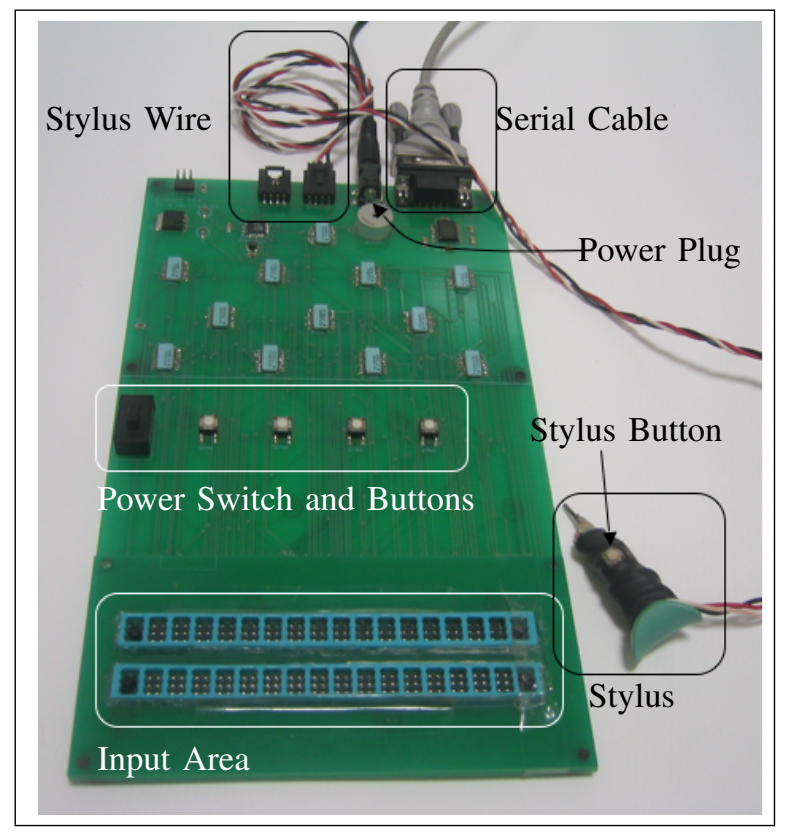

Fig. 3. The prototype E-Slate taken for field testing at the Mathru School for the Blind.

visual indicator for debugging early versions of the E-slate's software. Button 2 mutes the speaker so that more advanced students can use the E-slate without tonal feedback. Button 3 reverses the direction of the text, allowing students to choose between writing right to left (as is typical when writing with a standard slate and stylus) or left to right (which is the direction Braille is read in) ${ }^{2}$. Button 4 was included to allow an unprogrammed hardware input to the tutor software - it simply sends an acknowledgment to the computer when the button is pressed. The stylus connection port also contains two additional inputs for buttons located on the stylus. One button is placed on the stylus for students to indicate the completion of a character or word.

We initially considered several off-grid methods of powering the E-slate: solar cells, batteries powered by a solar charger, regular disposable batteries, and a hand crank to charge batteries. Unfortunately, except for disposable batteries which have a low but recurring cost, all of these options were more expensive than the E-slate itself! We decided that since the current iteration of the E-slate requires a computer powered by the electrical grid to be useful, expensive off-grid solutions should be explored at a later time. The E-slate is powered by an inexpensive AC/DC wall adapter which has an input range of 100 to $240 \mathrm{VAC}$ at $50 / 60 \mathrm{~Hz}$, and outputs up to 300 $\mathrm{mA}$ at $6 \mathrm{VDC}$. This input range is globally compatible with all electrical grid standards, and so the only adaptation that must be made to use it in different countries is to purchase an appropriate plug adapter.

\footnotetext{
${ }^{2}$ During our field study, we found that writing from left to right did not support transfer of writing skills to a regular slate and stylus and so disabled this feature.
}

\section{The Tutor Software}

The tutoring software was developed in close coordination with the teachers at the Mathru School for the Blind and was tailored to the needs of the students throughout the course of the field study. Together, we outlined three main stages of skill acquisition for the Braille student. The first step is to understand the concept of Braille and to emboss the six dots in a cell. The second step is to learn the unique combinations of dots that make up each letter and write the alphabet. The third step is to put letters together into words, put words together into sentences, and learn math symbols and punctuation. We created three different tutoring programs with emphasis on each of these skills and with capabilities to transition to the next skill.

Although Braille forms exist for many languages including the students' primary languages of Kannada and Tamil, they are taught Braille in English first because it is the standard approach and relatively simple (many Indic languages have more than $2^{6}$ characters and a single character may require more than one cell). Therefore our software tutor is currently limited to English Braille.

The software tutor receives input from the E-Slate regarding the learner's actions on the slate, where an "action" is either a contact between the stylus and the slate or a press of one of the five buttons. The tutor interprets these actions using a state machine and provides feedback tailored to the skill being learned.

\section{A. Second Standard Braille Writing Tutor.}

The second standard tutoring software meets the scaffolding needs of the beginner student learning the concept of six cells. Whenever the stylus is touched to the slate, the tutor speaks the position of the dot that was touched. This helps the student understand the cause and effect relationship between embossing on a slate and creating letters. It also teaches the spatial relationships between the different dots. The second standard tutor smoothly transitions to teaching and reinforcing the alphabet once the six-dots concept is learned: when the student presses the button on the stylus, the tutor will speak the letter written on the current cell. For simplicity, none of the other buttons on the E-Slate have any effect in this tutor. For the second standard tutor, we used a Mathru teacher's digitized voice for the dot and letter feedback. Firstly, there is a finite number of letters and positions so digitizing the feedback was feasible. Secondly and more importantly, we found that younger children using the second standard tutor may not be familiar with foreign accents and would feel more comfortable and learn faster if they heard their own teacher's voice.

\section{B. Third Standard Braille Writing Tutor.}

The third standard tutor partially meets the scaffolding needs of advanced students. The third standard tutor retains the position and letter feedback for reinforcement and spelling practice. It encourages speed by not requiring a student to press the stylus button to register a letter; instead, a letter 
is registered whenever the student transitions between cells. It additionally provides word feedback by keeping a character history and uses text-to-speech synthesis to speak the last word written when a student "double-clicks" the button on the stylus. For the TTS engine we use Cepstral's $囚$ female AmericanEnglish Callie voice [20] which they donated to the project. To augment the word feedback, we provide functionality both to erase and then correct previously-written letters and to spell the letters in the last word. This allows students to work on spelling as well as writing.

\section{Fourth Standard Braille Writing Tutor.}

The fourth standard tutor provides the remaining scaffolding for the advanced student. Here, we remove the position feedback as the learner is presumably familiar with the positions and finds such feedback cumbersome. This tutor additionally provides feedback on the last sentence written using the same text-to-speech engine. It also recognizes math symbols and punctuation which may require multiple cells per symbol, which have two symbols mapped to the same letter, or both. The tutor uses a decision tree to determine which symbol is intended.

\section{FIELD STUDY}

While at the Mathru school, we surveyed and evaluated students and teachers with regards to learning with, acceptance of, and usability of the tutor. Our target group was students in grades two and three as they had begun to learn Braille but had not yet mastered it, and so we would expect them to receive the greatest benefit from the Braille Tutor. This group consisted of six students in each grade for a total of twelve students. Ordinarily they had Braille class in four one-hour periods each week; for our study, they used the Braille Writing Tutor for forty minutes of the one hour and used a regular slate and stylus during the remaining twenty minutes. Although we focused our study on these twelve students, the tutors were constantly operational and we allowed any interested student or teacher to use them. As our goals for this pilot were to evaluate the feasibility and features of the Braille tutor, we forwent the opportunity to have a control group or crossover study in favor of allowing students unlimited and unstructured access to the tutor to measure their interest and responsiveness.

\section{A. Learning Gains}

We measured all twelve target students' proficiency in Braille once at the beginning of the study and once at the end and evaluated their improvement by assessing the skills learned; students were tested on a regular slate and stylus to ensure that student learning with the tutor transferred to regular slate and stylus despite the experiential differences between the two systems. Although it is difficult to attribute improvements solely to the tutor, we can determine its impact somewhat by understanding students' prior abilities. We tested how many cells the students could fill in with all six dots embossed (which we call the "six-dots test") and how many letters they could write (which we call the "alphabet test") in a fixed period of time; these are standard assessments the Mathru teachers use at this grade level to measure Braille writing proficiency. We also evaluated the number of mistakes made during the test; a mistake was defined as erroneously omitting or adding a dot or failing to leave a space between letters.

We can categorize the students into three groups based on a qualitative analysis of their pre- and post-trial test results. Four of the twelve students (call them Group A) demonstrated complete understanding of the Braille concept and could write the alphabet quickly and with few mistakes before we began the study. The tutor mainly provides advanced practice for these students. The second group (Group B) consisted of five students who lacked proficiency before the study but attained demonstrable proficiency by the end. The third group consisted of three students (call them Group C) who did not understand the concept of Braille and showed a significant lack of proficiency both before and after the study. We are interested in these last two groups to understand how the tutor may have helped those in Group B and why it did not help those in Group C.

Two of the five students in Group B (the group showing improvement) understood the concept of Braille before the study began but made frequent mistakes. At the end, they wrote significantly faster and made far fewer mistakes. Specifically, one student's abilities jumped from writing seven letters with four mistakes to writing thirteen letters perfectly, and the other student's abilities jumped from writing 23 letters with eight mistakes to writing 26 letters with one mistake. For these students, we suspect that the improvement was probably just the natural result of practice. Although the Braille Writing Tutor may have sped up their learning in comparison to using a regular slate because it increased their interest in writing, we cannot confidently make this claim without a control group.

The remaining three students in Group B made significant conceptual advances: in the first proficiency test they showed a distinct lack of understanding of Braille and were unable to emboss all six dots in a single cell. By the end of the study, two of the three students each wrote five letters with no mistakes and the other student completed the six-dots exercise in three cells. From our discussions with their teachers, we believe this is probably a direct result of getting immediate feedback from the tutor as these students had not demonstrated any understanding of Braille in the several months of instruction prior to the study. The case of one particular student highlights one way in which the tutor's instant feedback can produce a necessary conceptual advance. This student's writing usually consisted of a single cell with all the dots embossed, regardless of the assignment. It appeared that he had no conceptual understanding of Braille though he had been in Braille class for a few years. To the teachers' delight, this student began writing Braille as soon as he was asked to use the tutor. Apparently this student had always been writing every letter in the same cell, thus creating the completely embossed single cell. This was not evident to the student's teacher because the teacher herself was blind and could only feel the results of the writing on the paper; moreover, the student was unable to communicate well 


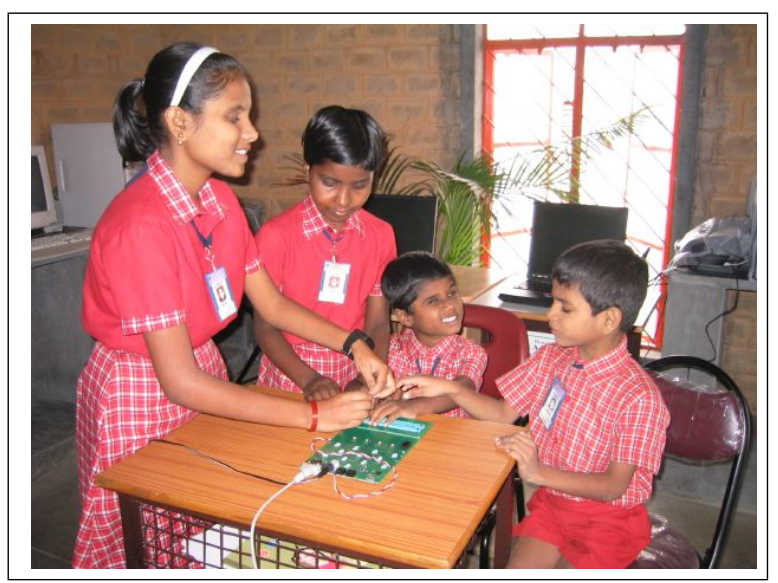

Fig. 4. Students at Mathru use the Braille Writing Tutor.

and explain what he was writing. Because the Braille Writing Tutor interprets letters after the student presses the button on the stylus, it did not matter that they were all in the same cell. Additionally, the teachers were able to hear the result of this student's writing immediately and soon realized that there was a gap in the student's understanding. In this way, the tutor acted as a diagnostic tool: it highlighted the student's unique difficulties and was able to provide insight to the teachers.

Although it is not clear why Group $\mathrm{C}$ did not benefit from the tutor (or from a month of Braille practice overall), our discussions with the teachers lead us to believe that they may not yet be developmentally ready to learn Braille. That is, we believe that members of Group $\mathrm{C}$ may have multiple disabilities/may need to develop basic social and personal skills.

\section{B. Tutor Acceptance}

The teachers' response to the tutor was very positive: they believe the tutor can help students overcome difficulties in writing Braille. Moreover, the teachers are eager to continue having the tutor in the classroom and have continued using them to this day. Their involvement in improving the software also highlighted their belief in the value of the tutor and they are further interested in bringing the tutor to other schools for the blind.

We evaluated the target students' attitude towards the tutor through an interview at the end of our six-week study. We asked them to agree or disagree with several statements:

1) I find the tutor useful.

2) I dislike using the tutor.

3) I prefer writing on a regular slate to writing on the ESlate.

4) I want to continue using the Braille Writing Tutor.

5) I think the tutor will help me in learning to write Braille.

To reduce the likelihood that the students would answer favorably simply out of respect or an eagerness to please, we explained to them that honesty was very important and had the teachers administer the survey when we were away from the school. Nearly all of the students (10/12) found the tutor

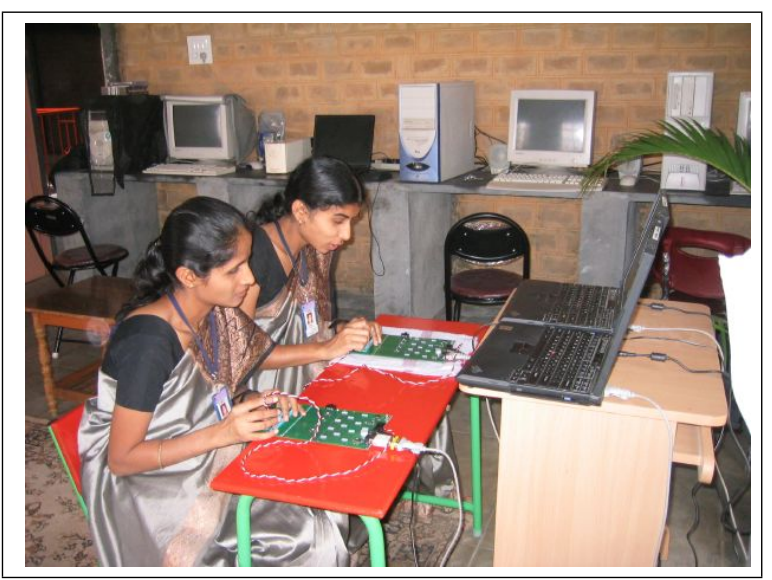

Fig. 5. The teachers use the Braille Writing Tutor at the tutor station we set up in the computer lab.

useful and believed it would help them in writing Braille; the remaining two students were in the second grade and had significant difficulty with the overall concept of Braille. However, all students strongly disagreed with the statement "I dislike using the tutor" and emphatically agreed with the statement "I want to continue using the tutor." Finally, nearly all students (10/12) preferred writing on the E-Slate to the regular slate, primarily because they enjoyed the interaction and the voice; the ones that did not were the youngest students who had difficulty finding the button on the stylus. While this indicates a positive response to the tutor, there is concern that students will adopt the E-Slate completely because it is easier to use and more interesting. We believe that this can be avoided in the school setting by having frequent exercises on a regular slate and stylus.

We evaluated the students' enthusiasm for writing Braille by observing which students wrote Braille outside of the Braille class period and the frequency of this use. We found that almost every student in the target group used the tutor outside of class at least once a week - only three of the students used it only during class. Interest varied from student to student: a few used the tutor on an almost daily basis while others used it once or twice a week. We also frequently found older students and even the teachers using the tutor in their spare time simply out of interest (see Figure 5). Though highly proficient in Braille, they simply enjoyed hearing the tutor speak their words and thoughts. However, students' interest in writing Braille extended only to using the tutor; it was rare to find students using the slate and stylus outside the classroom both before the introduction of the tutor and during the field trial.

\section{Usability Observations}

In addition to determining the learning gains and interest in the tutor, we observed how easy or difficult it was to setup and use by both teachers and students, asking ourselves the following questions:

- Hardware assembly. What are the minimum abilities required to assemble the hardware? How long does it 
take to learn how to assemble it? How long does it take to assemble?

- Software installation. What are the minimum abilities required to install and then start the software? How long does software installation take?

- Tutor use. How long does it take to learn how to start the tutor? What are the minimum abilities to start the tutor? How much and what type of instruction is required to learn how to use all the functionalities of the tutor?

We evaluated these features by observing both the teachers' and the students' use of the tutor. Hardware assembly involves plugging one end of the power adapter into a wall socket and inserting the other end into a socket on the E-Slate, connecting the serial cable between the E-Slate and the PC, and connecting the stylus to the E-Slate. We found that the blind teachers and students could only connect the power supply; connecting the serial cable and the stylus was impossible for them because the connectors are small and keyed. We trained a low-vision teacher with prior computer experience to successfully connect the serial cable and power in a single 30 minute training session but the stylus was simply too small to manipulate. Ultimately, this teacher was able to connect the power supply in under a minute but took 2-3 minutes to distinguish between the male and female ends of the serial cable, find the serial port on the back of the computer, and connect them. These difficulties highlight that we must completely redesign the hardware connection method to make assembly possible for our target group.

Software installation was also challenging as it involved a number of complex steps that required familiarity with advanced features of the Windows operating system. In the end, we installed all the software ourselves and successfully trained sighted teachers with significant prior computer experience. This highlights that software installation must also be redesigned significantly to install all components in a single step.

Nevertheless, once the components were installed and connected, the students could find the switch and turn on the E-Slate after only five minutes of training. We noticed two issues with students using the tutor: The smaller children had difficulty determining when the switch was set to the on position and when it was in the off position and both beginner and advanced students were initially afraid of receiving an electric shock from the electrical wire leading from the stylus to the slate. The older students ( $4^{t h}$ standard and higher) who had prior experience using a PC were able to start the Braille Writing Tutor software on their own with just minutes of instruction; younger children needed a teacher to do it for them.

At the end of the field study we also conducted a survey of the target group regarding usability. With the help of a teacher, we asked the children to agree or disagree with the following statements.

1) I found the Braille Writing Tutor difficult to use.

2) It took a long time to learn how to use the tutor.

3) I cannot understand the voice used by the tutor.
4) I find it difficult to distinguish between the buttons on the E-Slate.

Ten of the twelve students stated that it was easy to learn how to use the tutor and all the students could understand the voice used by the tutor even though the text-to-speech synthesizer used an adult female American voice. We suspect this may be because the students are accustomed to the JAWS screen reading software [21] which uses a similar male voice. Finally, roughly half of the students (5 of 12) found it difficult to distinguish between the buttons on the E-Slate. We believe that labeling the buttons and giving them unique shapes will alleviate this problem.

\section{SECOND DESIGN CyClE}

Our experiences at Mathru confirmed the feasibility of the Braille Tutor; students were able to use and learn from the tutor, and the tutors could be independently maintained by the school. Simultaneously, the study highlighted shortcomings of the current system and initiated a new design cycle, leading to refinements of both the E-slate and tutor.

\section{A. E-slate Changes}

Changes made to the E-slate fall into two categories; those made to improve the usability of the device, and those made because of a decision to link the slate more strongly to the computer. See table I for a summary of the major hardware changes and figure 6 for an image of the new E-slate.

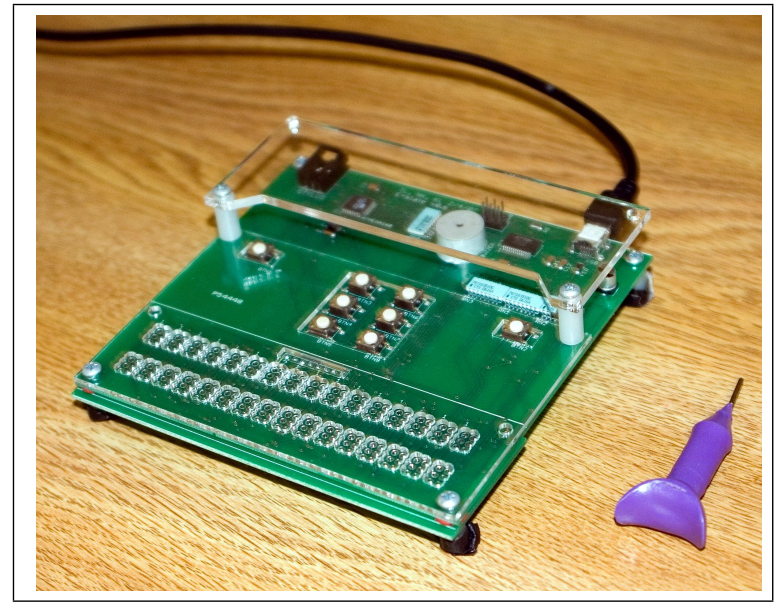

Fig. 6. Version 2 of the E-slate

1) Computer Dependence: The first E-slate had some standalone capability; without connecting to a computer, students could write on the slate and it would beep different notes depending on which Braille dot was sensed. After our experience at Mathru, we realized that this mode was unnecessary; students always used a computer with the slate, and computers were generally more available than we had expected. Although adding features is much more common during a design cycle, by removing standalone capability we were free to make a host of usability improvements to the E-slate, as well as lower the cost from approximately 40 USD to 30 USD if creating one hundred at a time. 
2) Usability Improvements: As we no longer need to power the slate separately from the computer, we changed the computer connection from serial to USB; USB devices can be powered directly from a computer. Removing the need for a separate power source lowers cost and partially addresses the difficulties teachers had installing the first Eslate. Additionally, since the E-slate is simply to be left plugged into the computer, the power on/off switch is no longer necessary the slate is now ready whenever the student wishes to use the tutor, eliminating the problems students had in finding the power switch. Finally, by moving to USB it is possible to attach multiple E-slates to a single computer, which allows for multi-player games and possibly reduces computer infrastructure costs; the number of E-slates which can be attached to a computer is limited only by the number of USB ports on the computer.

To address student concerns about safety, simplify the Eslate installation for teachers, and reduce the number of irreplaceable custom parts, we removed the wire connecting the slate to the stylus. Students are now able to use a normal, unmodified stylus with the E-slate; the enter button which used to be on the stylus has been moved to both sides of the slate. The other buttons have been replaced with a $2 \times 3$ button grid; these can be used by very young students who do not yet have the fine motor skills necessary to write Braille to practice the alphabet patterns. Although these six buttons have no additional current uses, by placing the buttons in a familiar grid older students can more readily resolve individual buttons should they be given functionality by future versions of the tutor.

\begin{tabular}{|l|l|l|}
\hline Feature & Version 1 & Version 2 \\
\hline $\begin{array}{l}\text { Computer Commu- } \\
\text { nication }\end{array}$ & Serial & USB \\
\hline $\begin{array}{l}\text { Button } \\
\text { Configuration }\end{array}$ & $\begin{array}{l}\text { Four equally spaced } \\
\text { buttons and 1 stylus } \\
\text { button }\end{array}$ & $\begin{array}{l}\text { button in } \\
\text { a Braille rell } \\
\text { arrangement and a } \\
\text { button on both the } \\
\text { left and right side } \\
\text { of the slate }\end{array}$ \\
\hline Stylus wired & Yes & No \\
\hline Power Source & Grid Power & $\begin{array}{l}\text { Computer through } \\
\text { USB }\end{array}$ \\
\hline $\begin{array}{l}\text { Braille cell configu- } \\
\text { ration }\end{array}$ & 32 cells in two rows & 32 cells in two rows \\
\hline Dust cover & No & Yes \\
\hline $\begin{array}{l}\text { Standalone capabil- } \\
\text { ity }\end{array}$ & Rudimentary & None \\
\hline
\end{tabular}

TABLE I

SUMMARY OF E-SLATE CHANGES BETWEEN VERSION 1 AND 2

\section{B. Tutor Improvements}

A major result of our pilot study at Mathru is that an electronically enhanced slate and stylus, used in concert with a computer, is a feasible method for teaching Braille. Having established feasibility, we focused our efforts on individualizing the tutor by having it adapt to student ability level and on developing automatic exercises for the student. To address the difficulties teachers had in setting up the software, we developed a new installation program. Finally, to expand the pool of people working on the tutor we developed a software library for application programmers which handles low level communications between the E-slate and computer.

1) Individualization: Chief among the educational difficulties faced by students in developing communities is the scarcity of Braille training and Braille teachers. Version two of the Braille tutor seeks to further ameliorate this problem by minimizing the amount of teacher attention required for learning. The software introduces a series of exercises forming a basic Braille writing curriculum; as before, the Tutor provides feedback to the student by narrating the student's actions. In addition, the tutor uses an English language 'Teacher voice'; this voice is distinct from the voice used to narrate the student's actions. The Teacher voice introduces exercises and gives feedback on how the student performed in each exercise. It also takes over narration of miscellaneous events such as Tutor mode changes and software errors. By introducing the Teacher voice, we allow interaction between the student and the Tutor to approximate interactions between the student and a personal teacher.

Students may begin practicing by selecting from six broad ability levels chosen at the start of each session: learning dots, practicing dots, learning letters, practicing letters, learning words, practicing words; these are analogous to the three grade levels used in version one of the tutor. The Tutor gathers information as the student attempts exercises in order to assign the most useful exercises to the student. For example, while the student is learning letters, each letter is monitored as an individual skill. If the student answers exercises involving the letter ' $a$ ' incorrectly, the Tutor will assign more exercises to practice ' $a$ '. If the estimated knowledge of the letter in question drops below a certain threshold, the Teacher voice reminds the student of how to write that letter by speaking a sequence of dots. Within each level, students are provided with exercises tailored to their unique needs.

2) Fast Installation: As help from sighted individuals was required for teachers to setup the Braille Tutor software, we developed a Braille Tutor installer program. The program provides audio feedback to the user at every decision point, with directions for which keyboard key to press to continue installation or cancel; the use of a mouse is not required. The new program cuts the installation time to under two minutes and launches the tutor at the end of the installation.

3) Extensible Software Design: The second version of the Braille Tutor software suite features a modular structure designed with future applications and development in mind. At its heart is a flexible software library that handles all lowlevel communication with the Tutor hardware and provides a variety of convenient interface facilities for the application programmer. The library encodes the user's interactions with the Tutor as a series of events of varying semantic complexity, ranging from the immediate insertion or removal of a stylus to the creation of an entire Braille character in one of the cells. Applications may poll a queue of selected events or be notified 
of them asynchronously through a callback interface.

The interface library tracks the state of the Braille Tutor hardware with an internal state machine model. By creating new state machine descriptions, the library can be adapted to future versions of the Braille Tutor hardware in a straightforward way. To the extent that hardware versions present compatible feature sets, the Braille Tutor library aims to provide a consistent software interface among them all, allowing maximum compatibility for applications.

\section{CONCLUSIONS}

Overall we believe the Braille Writing Tutor has great potential to inexpensively and effectively aid the education of a large number of blind students in the developing world. Our experiences thus far have been enormously positive, and we look forward to improving the tutor further and disseminating the technology to developing communities.

\section{A. Future Work}

1) Further Field Testing: In addition to continuing our work at Mathru, we are investigating a number of contacts at schools for the blind in Zambia, Russia, Trinidad and Tobago, the USA, and India with the goal of conducting field tests in the near future with the new version of the Braille Tutor. Unlike the feasibility study at Mathru, these studies aim to provide statistically significant evidence of the Braille Tutor's educational impact, and so we will run longer duration studies at multiple locations.

2) Hardware: In the long term, we believe a stand-alone device is essential to meet our design goals of usability in remote locations and among the very poor. This device would have the speaker and speech synthesis module and the tutor software embedded onto a mixed DSP/microcontroller. The tradeoff is that it may cost significantly more than our current E-Slate. Another possibility is to create a custom computer with multiple audio headphone outputs and a large number of USB ports. In this way it may be possible to run up to a dozen E-slates off of a single low cost computer, thus drastically reducing the cost of the computer-dependent tutoring system.

3) Software: We would like to tailor the speech feedback to the needs of the students. First, we hope to use ageand accent-appropriate English text-to-speech synthesizers to make the system more understandable and more engaging. Second, would like to extend the tutor to other languages and alphabets; while encoding the Braille script for other languages is relatively easy, this improvement does depend on the availability of at TTS engine in the language of interest. Last, we plan to take advantage of the ability to connect several E-slates to a single computer to create multi-player educational games.

\section{ACKNOWLEDGMENTS}

We would like to thank our partners at the Mathru School for the Blind for their partnership, for their constructive insights and feedback, and for hosting us during the field trial; this project would have been impossible without them. We also appreciate the advice and support of Manuela Veloso and Sarah Belousov. We would further like to thank Illah Nourbakhsh, Jack Mostow, Chris Atkeson, and Roberta Klatzky for their invaluable advice during the design phase of the project. We would like to thank Jeremy Stolarz for developing the Braille Tutor installation program.

\section{REFERENCES}

[1] World Health Organization, "Fact sheet 282: Magnitude and causes of visual impairment," World Health Organization, November 2004.

[2] E. Helander, Prejudice and dignity: an introduction to community-based rehabilitation. New York: UNDP, 1998.

[3] F. Schroeder, "Literacy: The key to opportunity," Journal of Visual Impairment and Blindness, pp. 290-293, June 1989.

[4] A. Nemeth, "Braille: The agony and the ecstasy," Braille Monitor, pp. 324-328, July 1998.

[5] Perkins, "Perkins brailler," Catalog of Products; Howe Press of the Perkins School for the Blind, 2006.

[6] Mathru School for the Blind, "Discussions with teachers and administrators," Unpublished, 2005.

[7] P. Brand and A. Schwittay, "The missing piece: Human-driven design and research in ict and development," Information and Communication Technologies and Development, 2006. ICTD '06, pp. 2-10, 2006.

[8] D. R. Millen, "Rapid ethnography: time deepening strategies for hci field research," Proceedings of the conference on Designing interactive systems: processes, practices, methods, and techniques, pp. 280-286, 2000.

[9] A. Cornwall and R. Jewkes, "What is participatory research?" Social Science and Medicine, vol. 41, pp. 1667-1676, 1995.

[10] K. Koedinger, J. Anderson, W. Hadley, and M. Mark, "Intelligent tutoring goes to school in the big city," International Journal of Artificial Intelligence in Education, vol. 9, pp. 30-43, 1997.

[11] J. Mostow, S. Roth, A. G. Hauptmann, and M. Kane, "A prototype reading coach that listens," in Proceedings of the Twelfth National Conference on Artificial Intelligence (AAAI-94), 1994.

[12] T. H. Bunnell, D. M. Yarrington, and J. B. Polikoff, "STAR: articulation training for young children," in International Conference on Spoken Language Processing, 2000.

[13] G. Weber and P. Brusilovsky, "Elm-art: An adaptive versatile system for web-based instruction," International Journal of Artificial Intelligence in Education, vol. 12, no. 4, pp. 351-384, 2001.

[14] F. L. Peaco, "Braille literacy: Resources for instruction, writing equipment, and supplies," NLS Reference Circulars, 2004.

[15] J. Chamot, "Electronic braille tutor teaches independence," National Science Foundation Press Release, 2006.

[16] C. S. Lahiri A. and B. A, "Sparsha : A comprehensive indian language toolset for the blind." Proceedings of the 7th International ACM SIGACCESS conference on Computers and Accessibility, pp. 114-120, 2005.

[17] [Online]. Available: http://www.webelmediatronics.in/brlads/devices.htm

[18] [Online]. Available: http://www.techbridgeworld.org

[19] Atmel, "Atmega88 datasheet," Atmel Corp., 2006. [Online]. Available: http://www.atmel.com/dyn/resources/prod_documents/doc2545.pdf

[20] [Online]. Available: http://cepstral.com

[21] [Online]. Available: http://www.freedomscientific.com/fs_products/ software_jaws.asp 